\title{
Prevalência de Doença Periodontal em pacientes com Insuficiência Renal Crônica internados em UTI pública em Manaus/AM
}

Prevalence of Periodontal Disease in Patients with Chronic Kidney Failure admitted to a public ICU in Manaus/AM

Prevalencia de enfermedad periodontal en pacientes con insuficiencia renal crónica ingresados en una UCI pública en Manaus/AM

Recebido: 23/06/2021 | Revisado: 29/06/2021 | Aceito: 01/07/2021 | Publicado: 15/07/2021

\author{
Danielle Lima Torres \\ ORCID: https://orcid.org/0000-0002-3211-2547 \\ Universidade Paulista, Brasil \\ E-mail: danny_limma_df@hotmail.com \\ Nely Cristina Medeiros Caires \\ ORCID: https://orcid.org/0000-0003-3094-4064 \\ Universidade Paulista, Brasil \\ E-mail: nelycaires@gmail.com \\ Leonardo de Resende Ferreira \\ ORCID: https://orcid.org/0000-0002-8491-6416 \\ Universidade Paulista, Brasil \\ E-mail: leoabo@hotmail.com
}

\begin{abstract}
Resumo
A inter-relação entre Doença Periodontal (DP) e Insuficiência Renal Crônica (IRC) é inevitável, pois pacientes com IRC apresentam características intrabucais que predispõem e/ou agravam a DP como: xerostomia, periodontite, reabsorção óssea, cálculo dentário e grande acúmulo de biofilme dental. O objetivo desse estudo foi avaliar a prevalência da DP em pacientes com IRC internados em uma UTI pública em Manaus/AM. Todos os pacientes internados que tinham no mínimo 6 dentes, um por sextante, eram elegíveis para participar desse estudo. O Exame Periodontal Simplificado foi realizado através da medida da profundidade de sondagem para detecção de bolsa periodontal em 3 pontos: médio vestibular, mesio vestibular e disto vestibular. Dos 30 pacientes incluídos na análise, $63,3 \%$ eram do gênero masculino, vinte e oito $(93,3 \%)$ apresentavam doença periodontal, enquanto apenas dois $(6,7 \%)$ tinham o periodonto saudável. Uma forma generalizada de periodontite foi observada em 21 pacientes (75\%). E levando-se em consideração a severidade da periodontite, apenas 8 dos pacientes $(28,6 \%)$ tinham o grau leve da doença. Concluiu-se que, nesta amostra, a prevalência da DP em pacientes com IRC internados na UTI analisada é elevada, com predominância de uma forma generalizada de periodontite. Desta forma, a IRC parece não ser por si só, o único fator desencadeante das altas taxas de mortalidade que acometem os pacientes internados em UTI, o que comprova a importância e necessidade do cirurgião-dentista em equipe multiprofissional em ambiente hospitalar.
\end{abstract}

Palavras-chave: Insuficiência renal crônica; Doença periodontal; Unidade de Terapia Intensiva; Cirurgião-dentista.

\begin{abstract}
The interrelation between Periodontal Disease (PD) and Renal Insufficiency Chronic (RIC) is unavoidable, as patients with RIC have intrabuccal characteristics that predispose and / or aggravate PD such as: xerostomia, periodontitis, bone resorption, dental calculus and large accumulation of dental biofilm. The aim of this study was to assess the prevalence of PD in patients with RIC admitted to a public ICU in Manaus / AM. All in patients who had at least 6 teeth, one per sextant, were eligible to participate in this study. The Simplified Periodontal Examination was performed by measuring the depth of the probe to detect the periodontal pocket at 3 sites: mediovestibular, mesiovestibular and distovestibular. Of the 30 patients included in the analysis, $63.3 \%$ were male, twenty-eight (93.3\%) had periodontal disease, while only two $(6.7 \%)$ had a healthy periodontium. A generalized form of periodontitis was seen in 21 patients (75\%). And taking into account the severity of periodontitis, only 8 of the patients $(28.6 \%)$ had a mild degree of the disease. It was concluded that, in this sample, the prevalence of PD in patients with RIC admitted to the ICU is high, with a predominance of a generalized form of periodontitis. Thus, RIC does not seem to be in itself the only triggering factor for the high mortality rates that affect patients admitted to the ICU, which proves the importance and need of the dentist in a multidisciplinary team in a hospital environment.
\end{abstract}

Keywords: Renal insufficiency chronic; Periodontitis; Intensive Care Units; Dentists. 


\section{Resumen}

La interrelación entre Enfermedad Periodontal (EP) e Insuficiencia Renal Crónica (IRC) es inevitable, ya que los pacientes con IRC tienen características intraorales que predisponen y / o agravan la EP, como xerostomía, periodontitis, resorción ósea, cálculos dentales y gran acumulación de biofilm dental. El objetivo de este estudio fue evaluar la prevalencia de EP en pacientes con ERC ingresados en una UCI pública en Manaus / AM. Todos los pacientes hospitalizados que tenían al menos 6 dientes, uno por sextante, fueron elegibles para participar en este estudio. El examen periodontal simplificado se realizó midiendo la profundidad de sondaje para la detección de bolsas periodontales en 3 puntos: vestibular medio, mesiobucal y distobucal. De los 30 pacientes incluidos en el análisis, el $63,3 \%$ eran hombres, veintiocho $(93,3 \%)$ tenían enfermedad periodontal, mientras que solo dos $(6,7 \%)$ tenían un periodonto sano. Se observó una forma generalizada de periodontitis en 21 pacientes $(75 \%)$. Y teniendo en cuenta la gravedad de la periodontitis, solo 8 de los pacientes $(28,6 \%)$ tenían enfermedad leve. Se concluye que, en esta muestra, la prevalencia de EP en pacientes con IRC ingresados en la UCI analizada es alta, con predominio de una forma generalizada de periodontitis. Así, la IRC por sí sola no parece ser el único factor desencadenante de las altas tasas de mortalidad que afectan a los pacientes ingresados en UCI, lo que demuestra la importancia y necesidad de contar con odontólogos en un equipo multidisciplinar en el ámbito Hospitalar.

Palabras clave: Insuficiencia renal crónica; Enfermedad periodontal; Unidad de Cuidados Intensivos; Cirujano dentista.

\section{Introdução}

Uma das inúmeras doenças sistêmicas observadas em pacientes internados em UTI (Unidade de Terapia Intensiva) é a Insuficiência Renal Crônica (IRC), que consiste em lesão renal e perda progressiva e irreversível da função dos rins. A IRC se tornou um grande problema de saúde pública no mundo (Lima et al., 2014).

As principais causas de IRC são: Diabetes Mellitus, Nefrosclerose Hipertensiva e as Glomerulonefrites Crônicas. As principais causas de óbitos de pacientes com IRC são as doenças cardiovasculares, como acidente vascular cerebral e a doença coronariana. A DP também tem sido associada com doenças ateroscleróticas, pois os processos micro inflamatórios estão vinculados com a progressão dos processos aterogênicos devido à liberação de mediadores químicos inflamatório pela corrente sanguínea, o que aumenta o risco de complicações decorrentes da aterosclerose (Kim et al., 2017)

A inter-relação entre DP e IRC é inevitável, pois as alterações do periodonto podem ser provocadas principalmente pela carga inflamatória sistêmica e a baixa imunidade causadas pela IRC; entretanto, em situação inversa, a DP tem sido relacionada como fator de risco à IRC devido à disseminação sistêmica de microrganismos e produtos da inflamação periodontal (Deschamps-Lenhardt et al., 2019).

A maioria dos pacientes renais crônicos não tem como prioridade a higiene bucal e o tratamento dos dentes, pois sua maior preocupação é com a sua saúde sistêmica. Além disso, a dificuldade de acesso ao tratamento dentário público ou privado por estes pacientes contribui para o aparecimento de doenças periodontais, o que representa foco de infecção nos pacientes renais crônicos, e pode, inclusive, contraindicar o transplante renal (Araujo et al., 2016; Castro et al., 2020).

Diante do exposto acima, observa-se que a inclusão do cirurgião dentista em um ambiente hospitalar é necessária para oferecer suporte especializado em diagnósticos das modificações bucais associadas ao tratamento médico. Suas ações são solicitadas em procedimentos emergenciais diante de traumas dentários, em tratamentos preventivos para impossibilitar o agravamento sistêmico ou surgimento de uma infecção hospitalar advinda da cavidade bucal, e tratamentos curativos e restauradores de adequação do meio bucal, favorecendo maior conforto e saúde para o paciente (Blum et al., 2018; Silva et al., 2020)

Diante das evidências de correlação prejudicial entre a DP e IRC e a alta prevalência das duas doenças nas UTIs, o objetivo deste trabalho foi realizar um estudo sobre a prevalência da Doença Periodontal em pacientes com Insuficiência Renal na Unidade de Terapia Intensiva de um Hospital público em Manaus/AM além de reforçar a importância da atuação do cirurgião-dentista no ambiente hospitalar. 


\section{Metodologia}

Trata-se de um estudo transversal, quali-quantitativo (Estrela, 2018), que avaliou a associação entre Doença Periodontal e Insuficiência Renal Crônica, em um total de 30 pacientes, de ambos os gêneros, internados na UTI de um hospital público em MANAUS/AM. Para que o indivíduo fosse incluído na amostra deveria obedecer aos seguintes critérios: apresentar quadro sistêmico de Insuficiência Renal Crônica submetido ou não à hemodiálise e possuir, no mínimo, um total de 6 (seis) elementos dentais em sua cavidade bucal. Os pacientes edêntulos e os que não possuíam pelo menos um dente por sextante foram excluídos do estudo.

Os dados foram coletados após a assinatura de Termo de Consentimento Livre e Esclarecido (TCLE) pelos pacientes ou responsáveis. Os dados foram coletados por um único pesquisador.

O trabalho foi previamente submetido e aprovado pelo Comitê de Ética e Pesquisa (CEP), pelo parecer $\mathrm{n}^{\circ}$ 30513014.3.0000.5512.

Foi realizada inicialmente, anamnese, com o objetivo de avaliar a história médica e odontológica dos pacientes, seguida do exame clínico periodontal. Como instrumento para a coleta dos dados, foi utilizado um formulário contendo os seguintes dados: gênero, idade, total de elementos dentais presentes na cavidade oral, Condição Sistêmica. Para avaliar o índice de sangramento e a profundidade de sondagem foi utilizada ficha de Exame Periodontal de registro periodontal simplificado (PSR).

Depois de assinado o TCLE pelos pacientes e examinado o prontuário clínico, foi realizada a Avaliação Periodontal Simplificada (PSR) através da profundidade de sondagem para detecção de bolsas periodontais em 3 pontos: vestibular, mesiovestibular e distovestibular. Posteriormente, classificou-se a periodontite em localizada, com extensão $<$ ou $=3$ sextantes afetados, e generalizada quando a extensão abrangia mais de 3 sextantes. Quanto à severidade, classificou-se como leve na presença do código 2, moderada na presença do código 3 e severa na presença do código 4.

A coleta de dados foi realizada através de sonda Periodontal WHO-PSR (sonda milimetrada) da marca Golgran® (Figuras 1). A Figura 2 mostra a sondagem do sulco gengival, durante a coleta dos dados.

Figura 1: Sonda Periodontal WHO-PSR.

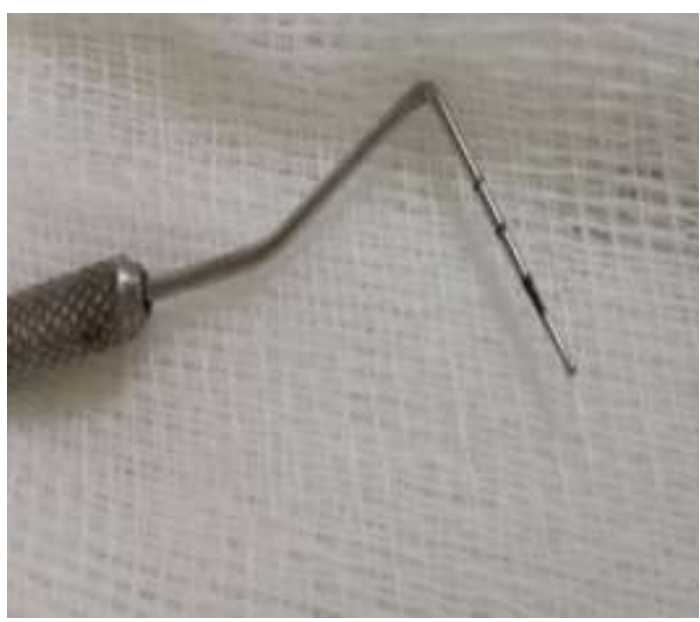

Fonte: Autores.
Figura 2: Sondagem do sulco gengival.

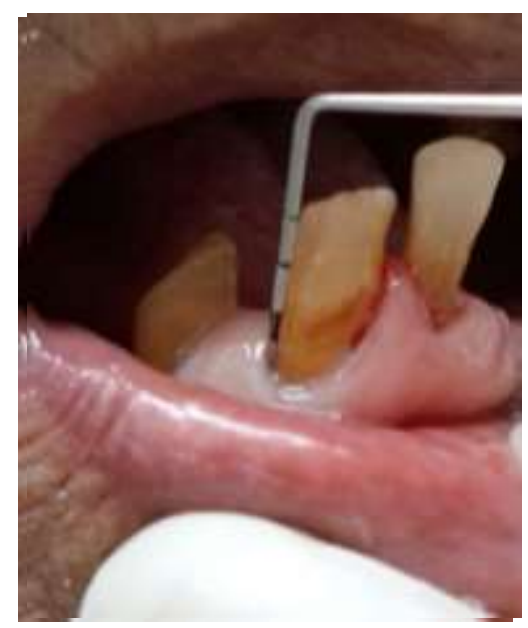

Fonte: Autores.

Após a coleta de dados, foi realizada a análise estatística descritiva, com caracterização da amostra estudada por meio de medidas de tendência central e dispersão, além de frequências simples e relativas, utilizando-se o programa Microsoft Excel®. 


\section{Resultados}

O total de pacientes internados na UTI do Hospital público, objeto do estudo, que apresentavam diagnóstico de IRC era de 45 pacientes. Deste total, apenas 30 pacientes depois de avaliados os critérios de inclusão, estavam aptos e consentiram em participar do estudo, após ler e assinar o TCLE.

Quanto ao gênero e idade (Tabela 1), a maioria dos pacientes eram do gênero masculino $(63,4 \%)$ e a idade prevalente foi entre 70 e 90 anos de idade (35\%). Quanto ao número de dentes presentes na cavidade oral, nota-se que 76,6\% dos participantes possuíam entre 6 a 17 elementos dentais.

Tabela 1 - Dados descritivos dos pacientes quanto a idade e gênero e total de elementos dentais.

\begin{tabular}{lll}
\hline \multicolumn{1}{c}{ VARIÁVEL } & \multicolumn{2}{c}{ IRC $\mathbf{n = 3 0}$} \\
\cline { 2 - 3 } & \multicolumn{1}{c}{$\mathbf{n}$} & \% \\
\hline Gênero & & \\
Feminino & 11 & 36,6 \\
Masculino & 19 & 63,4 \\
Idade & & \\
$\mathbf{1 8 - 3 0}$ & & \\
$\mathbf{3 0 - 5 0}$ & 6 & 25 \\
$\mathbf{5 0 - 7 0}$ & 3 & 10 \\
$\mathbf{7 0 - 9 0}$ & 10 & 30 \\
& 11 & 35 \\
Total de dentes presentes & & \\
$\mathbf{6}$ a 17 & \multicolumn{2}{c}{} \\
$\mathbf{1 7}$ a 32 & 23 & 76,6 \\
\hline
\end{tabular}

Fonte: Autores.

No Gráfico 1, nota-se que apenas 2 pacientes apresentaram ausência de doença periodontal.

Gráfico 1 - Condição Periodontal dos pacientes com IRC.

IRC X PRESENÇA DE DP

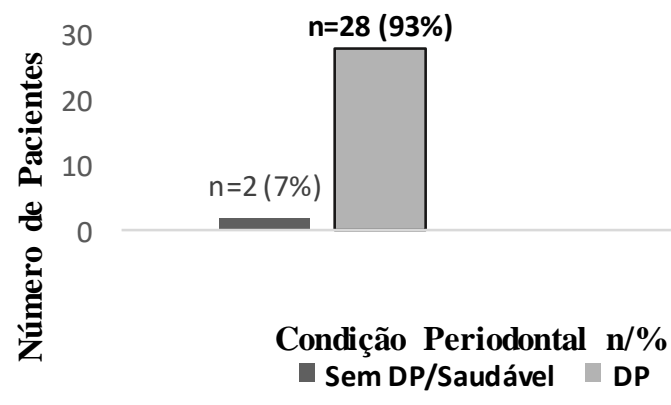

Fonte: Autores.

$\mathrm{Na}$ avaliação da gravidade da Doença Periodontal entre os 28 pacientes com DP, notamos na Tabela 2, que a maioria dos pacientes $(46,4 \%)$ apresentaram DP de forma Moderada e $25 \%$ apresentaram a doença na forma severa da doença, conforme descrito na Tabela 2. 
Tabela 2 - Severidade da Doença Periodontal.

\begin{tabular}{lcc}
\hline Classificação & n & Percentual (\%) \\
\hline Leve & 8 & 28,6 \\
Moderada & 13 & 46,4 \\
Severa & 7 & 25 \\
Total & 28 & 100 \\
\hline
\end{tabular}

Fonte: Autores.

A Tabela 3, diz respeito à extensão da Doença Periodontal. A forma generalizada da doença mostrou-se mais prevalente, com $21(75 \%)$ pacientes acometidos.

Tabela 3 - Classificação quanto a Localização da DP.

\begin{tabular}{lcc}
\hline Classificação & n & Percentual (\%) \\
\hline Localizada & 7 & 25 \\
Generalizada & 21 & 75 \\
Total & 28 & 100 \\
\hline
\end{tabular}

Fonte: Autores.

Ao se relacionar a severidade da doença com sua localização, observamos que quase metade (43\%) dos pacientes apresentaram a forma generalizada e severa da DP (Gráfico 2).

Gráfico 2 - Associação entre Severidade e localiação da DP.

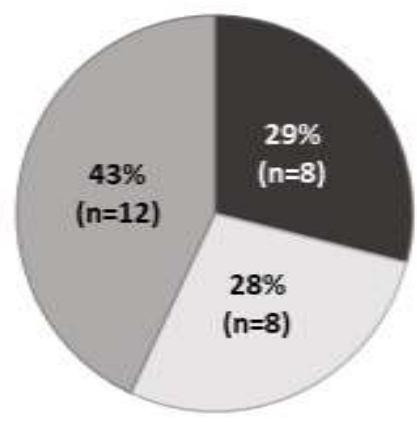

\author{
meneralizada e Severa \\ Localizada e Leve \\ Generalizad a e Moderada
}

Fonte: Autores.

\title{
4. Discussão
}

Pacientes renais crônicos apresentam alterações da saúde bucal, tais como: elevado acúmulo de placa e cálculo dentário, diminuição da secreção salivar, hálito urêmico, palidez da mucosa bucal (Fréo, 2014; Quadrelli, Sousa, 2019). Tal afirmação foi comprovada em nosso estudo. Os resultados mostraram que, nesta amostra a prevalência da Doença Periodontal em pacientes com Doença Renal Crônica é elevada. Dos 30 pacientes participantes da pesquisa, 93\% apresentaram DP.

Menos da metade dos avaliados possuíam entre 6 a 17 elementos dentais na boca, representa um elevado índice CPOD com muitas perdas dentais. Estes achados mostram que há necessidade de tratamento/reabilitação destes pacientes, além da adoção de medidas preventivas. Além disso, pacientes que apresentavam menor quantidades de unidades dentárias também demonstraram presença de Doença periodontal (Gonçalves et al., 2011).

Vários são os fatores que podem levar a esta perda dental tão acentuada entre os pacientes avaliados. Dentre elas destaca-se o fator psicossocial, como a depressão que ocorre nesses pacientes. Isto faz com que não haja mais interesse pelo 
autocuidado e consequentemente a saúde bucal é deixada de lado, ocorrendo então um avanço gradativo das doenças orais, principalmente as periodontopatias, por causa da baixa qualidade de higiene oral (Norte, 2017)

Quanto ao gênero, a maioria dos pacientes com DRC eram do gênero masculino. Esse achado está de acordo com outros estudos que demonstram que os homens têm sido mais acometidos por doenças crônicas, incluindo as renais. Os indivíduos do gênero masculino julgam-se menos vulneráveis, o que contribui para que ele cuide menos da própria saúde e se exponha mais às situações de risco (Ribeiro et al., 2014; Santos et al., 2018).

Pacientes portadores DRC apresentam também características sistêmicas que alteram e prejudicam o periodonto da cavidade oral. Isso está de acordo com os achados deste estudo, onde 93\% possuem doença periodontal. Este fato pode estar relacionado com a anemia, que provoca uma maior exacerbação da resposta inflamatória da gengiva ao biofilme presente. A uremia também favorece o aumento da ureia salivar e consequentemente alteração do $\mathrm{pH}$ do biofilme dental, ocasionando maiores depósitos de cálculo dental. Quanto mais sítios ativos e quanto maior for o tempo de exposição à DP, maior é o risco das infecções a distância (Lacerda et al., 2015).

A presença de pacientes portadores de Doença Periodontal em nosso estudo demonstra que além da higienização estar deficiente, a alteração no metabolismo do Ca e P e o PTHS, que atuam no metabolismo do tecido ósseo, interfere na sua modelação e promove uma maior reabsorção óssea, podendo contribuir para uma maior severidade da DP em pacientes que possuem IRC, dados estes que estão em consonância com os achados de outros autores (Kovesdy, 2010; Almeida, 2011)

A maior prevalência de uma forma generalizada de DP no grupo de pacientes demonstrados neste estudo não está de acordo com os dados epidemiológicos do SB Brasil (2004).

Uma alternativa que pode melhorar o quadro clínico de um paciente com Doença Renal Crônica e que possui doença Periodontal, seria a terapia periodontal tradicional. Este tratamento compreende raspagem periodontal e manutenção destes pacientes, visando que a infecção presente no periodonto seja controlada. Estes procedimentos simples e rotineiros podem diminuir em até 3 vezes a PCR no organismo deste paciente (Kim et al., 2017; Spezzia, 2021).

Estando a DP relacionada com a DRC, é necessário se obter e manter um excelente controle de higiene bucal, para que as periodontites não se tornem fatores agravantes do estado geral do paciente renal crônico (Almeida et al., 2009; Deschamps-Lenhardt et al., 2019).

O acompanhamento odontológico por um cirurgião-dentista, tem como objetivos a avaliação da condição bucal dos pacientes, levando em consideração a presença de biofilme na cavidade oral, doença periodontal, presença de cáries, lesões bucais infectadas precursoras de infecções virais e fúngicas sistêmicas, lesões traumáticas. A possibilidade de realizar um plano de tratamento odontológico adequado para o paciente internado em ambiente hospitalar, possibilita o restabelecimento e a manutenção da saúde bucal deste paciente (Gomes et al., 2014).

\section{Considerações Finais}

Este trabalho nos permitiu concluir que a prevalência da Doença Periodontal em pacientes com Doença Renal Crônica, foi elevada. Sendo assim, há necessidade da inclusão de um Cirurgião Dentista como um suporte no diagnóstico e no tratamento das alterações bucais como coadjuvante na terapêutica médica. Deve ser incluído na terapêutica médica dos pacientes com Doença Renal Crônica o Tratamento Odontológico e Periodontal tradicional, para proporcionar a estes pacientes uma melhora em seu quadro sistêmico. Esperamos que trabalhos futuros continuem apontando as melhorias na condição sistêmica de pacientes internados em UTIs que possuem o cirurgião-dentista integrado à equipe multiprofissional, além de despertar nos gestores de hospitais públicos e privados, a inclusão deste profissional, com vistas a prevenção, promoção de saúde e tratamento dos pacientes ali internados, já que comprovado está o papel da saúde bucal na condição sistêmica do paciente 


\section{Referências}

Almeida, D. C. (2011). Status periondontal em pacientes portadores de doença renal crônica terminal em terapia substitutiva de hemodiálise. $125 \mathrm{f}$. Dissertação (Mestrado em Clínica Odontológica) - Universidade Federal Fluminense - 2011.

Almeida, D. C., Machado, W. A. S., Tostes, F. R. V., \& Santana, R. B. (2009). Inter-relação entre Insuficiência renal crônica em hemodiálise e doença periodontal. Jornal Brasileiro de Medicina, 96(6): 16-19.

Araújo, L. F., Castelo Branco, C. M. C., Rodrigues, M. T. B., Cabral, G. M. P., \& Diniz, M. B. (2016). Manifestações bucais e uso de serviços odontológicos por indivíduos com doença renal crônica. Revista da Associação Paulista de Cirurgioes Dentistas, 70(1):30-6.

Bayraktar, G., Kurtulus, I., Kazancioglu, R., Bayramgurler, I., Cintan, S., Bural, C., Besler, M., Trablus, S., Issever, H., Aysuna, N., Ozkan, O., \& Yildiz, A. (2009). Effect of educational level on oral health in peritoneal and hemodialysis patients. International Journal of Dentistry, $2009: 1-5$.

Blum, D. F., Silva, J. A. S., Baeder, F. M., \& Bona, A. D. (2018). A atuação da Odontologia em unidades de terapia intensiva no Brasil. Revista Brasileira de Terapia Intensiva, 30(3):327-332.

Brasil. Ministério da Saúde. Projeto SB 2000. Condições de Saúde Bucal da População Brasileira. Relatório Final. Brasília, DF, 2004.

de Castro, G. D., Cabreira, N. T., \& Santi, S. S. (2020). Associação entre Periodontite e Doença Renal Crônica - Revisão Sistemática. Brazilian Journal of Periodontology, 29(3):99-112.

Deschamps-Lenhardt, S., Martin-Cabezas, R., Hannedouche, T., \& Huck, O. (2019). Association between periodontitis and chronic kidney disease: Systematic review and meta-analysis. Oral Disease, 25(2):385-402.

Estrela, C. (2018). Metodologia Científica: ciência, ensino, pesquisa. (3a ed.), Artes Médicas.

Fréo, B. (2014). Manifestações bucais em pacientes portadores de insuficiência renal crônica - correlações com níveis de ansiedade e depressão, percepção da saúde oral e qualidade de vida 104f. Tese (Doutorado Programa de Pós-Graduação em Odontologia) Faculdade de Odontologia da universidade de São Paulo.

Gomes, S. F., \& Esteves, M. C. L. (2012). Atuação do cirurgião-dentista na UTI: um novo paradigma. Revista Brasileira de Odontologia, 69 (1): $67-70$.

Gonçalves, E. C., D. L. F. L., de Albuquerque, S. H. C., de Carvalho, J. A., Cariri, T. F. A., de Oliveira, C. A. C. (2011). Avaliação da perda de inserção dentária em pacientes com doença renal crônica em hemodiálise. Brazilian Journal of Nephrology. 33 (3): 291-294.

Kim, Y. J., Moura, L. M., Caldas, C. P., Perozini, C., Ruivo, G. F., \& Pallos, D. (2017). Einstein, 15(2):173-7.

Kovesdy, C. P. (2010). Rate of kidney function decline associates with increased risk of death. Journal of the American Society of Nephrology, 21(11):18141816.

Lacerda, M. C. S. R., Viana, K. B., Dores, D. F., Bessa-Nogueira, R. V., \& Ribeiro, C. M. B. (2015). Caracterização da saúde bucal de indivíduos renais crônicos aptos a transplante. Revista de Odontologia UNESP, 44(5): 292-298.

Lima, M. C. P. S., Vieira, G. G., \& Duarte, D. A. (2014). Avaliação da condição periodontal de pacientes sob hemodiálise na cidade de Imperatriz-MA. Arquivos Médicos dos Hospitais e da Faculdade de Ciências Médicas da Santa Casa de São Paulo, 59(3):117-120.

Norte, A. E. O. (2017). Qualidade de vida relacionada à saúde bucal e depressão em pacientes com doença renal crônica submetidos à hemodiálise. 57 f. Dissertação (Mestrado em Saúde da Família) - Universidade Federal do Ceará, Sobral, 2017.

Quadrelli, J. B. S., \&, C. O. (2019). Manifestações Bucais e o Manejo Odontológico em Pacientes com Doença Renal Crônica. Revista da Jopic, 2(4):104114.

Rabelo, G. D., Queiroz, C. I., \& Santos, P. S. S. (2010). Atendimento odontológico ao paciente em unidade de terapia intensiva. Arquivos Médicos dos Hospitais e da Faculdade de Ciências Médicas da Santa Casa de São Paulo, 55(2):67-70.

Ribeiro, I. P., Pinehiro, A. L. S., Soares, A. L. A., \& Santos, N. F. M. (2014). Perfil epidemiológico dos portadores de insuficiência renal crônica submetidos à terapia hemodialítica. Enfermagem em Foco, 5(3/4): 65-69.

Santos, K. K., Lucas, T. C., Glória, J. C. R., Pereira Júnior, A. C., Ribeiro, G. C., \& Lara, M.O. (2018). Perfil Epidemiológico De Pacientes Renais Crônicos Em Tratamento. Revista de Enfermagem UFPE on line, 12(9):2293-300.

Silva, E. A., Silva, A. B. S., Machado, I. F., Irmão Bisneto, J. S. L., Santos, I. I. S., \& Cota, A. L. S. (2020). A importância da atuação do cirurgião dentista na unidade de terapia intensiva: revisão integrativa. Research, Society and Development, 9(6): e164962011.

Spezzia, S. (2021). Doença renal crônica e doenças periodontais. Odonto, 29(56): 1-8. 